\title{
A Fabry-Pérot interferometer with wire- grid polarizers as beamsplitters at terahertz frequencies
}

Cite as: Rev. Sci. Instrum. 89, 035116 (2018); https://doi.org/10.1063/1.5018400

Submitted: 06 December 2017 . Accepted: 10 March 2018 . Published Online: 29 March 2018

H. Harrison (D, A. J. Lancaster, I. V. Konoplev (D, G. Doucas, A. Aryshev (D), M. Shevelev, N. Terunuma (D), J. Urakawa (D), and P. C. Huggard (iD)

\section{ARTICLES YOU MAY BE INTERESTED IN}

Observation of coherent Smith-Purcell and transition radiation driven by single bunch and micro-bunched electron beams

Applied Physics Letters 112, 053501 (2018); https://doi.org/10.1063/1.5009396

Metasurface for multi-channel terahertz beam splitters and polarization rotators

Applied Physics Letters 112, 17111 (2018); https://doi.org/10.1063/1.5028401

Contact angle measurement with a smartphone

Review of Scientific Instruments 89, 035117 (2018); https://doi.org/10.1063/1.5022370

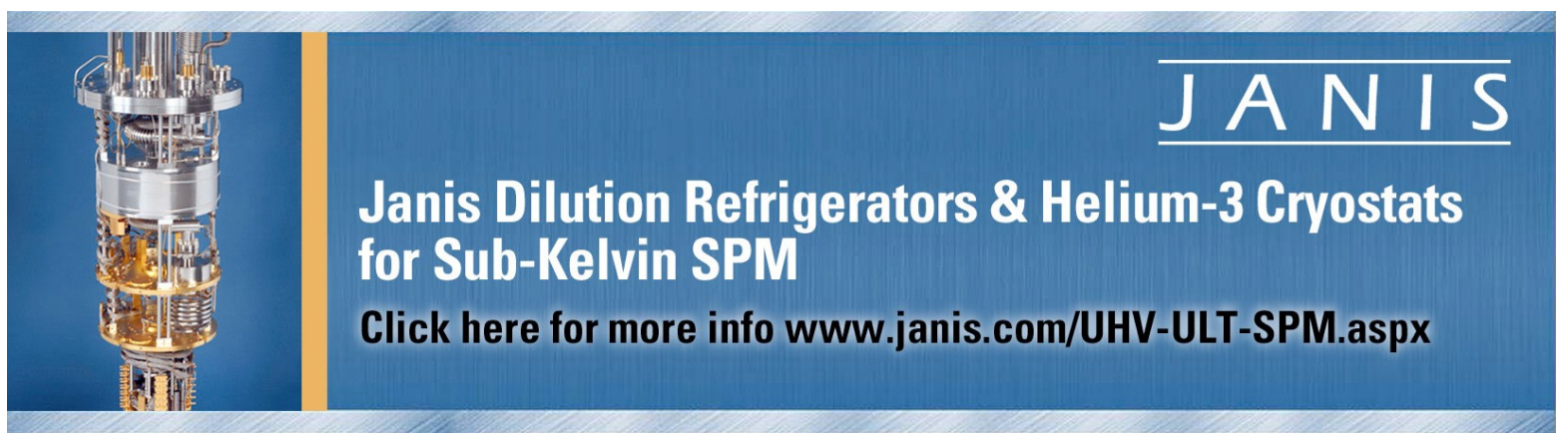




\title{
A Fabry-Pérot interferometer with wire-grid polarizers as beamsplitters at terahertz frequencies
}

\author{
H. Harrison, ${ }^{1, a)}$ A. J. Lancaster, ${ }^{1, b)}$ I. V. Konoplev,${ }^{1}$ G. Doucas, ${ }^{1}$ A. Aryshev, ${ }^{2}$ M. Shevelev,,${ }^{2,3}$ \\ N. Terunuma, ${ }^{2}$ J. Urakawa, ${ }^{2}$ and P. G. Huggard ${ }^{4}$ \\ ${ }^{1}$ The John Adams Institute, Department of Physics, University of Oxford, Oxford OX1 3RH, United Kingdom \\ ${ }^{2}$ KEK, High Energy Accelerator Research Organization, 1-1 Oho, Tsukuba, Ibaraki 305-0801, Japan \\ ${ }^{3}$ Tomsk Polytechnic University, Lenin Avenue 30, Tomsk 634050, Russian Federation \\ ${ }^{4}$ MMT Group, RAL Space, STFC Rutherford Appleton Laboratory, Didcot OX11 OQX, United Kingdom
}

(Received 6 December 2017; accepted 10 March 2018; published online 29 March 2018)

The design of a compact Fabry-Pérot interferometer (FPi) and results of the experimental studies carried out using the device are presented. Our FPi uses freestanding wire-grid polarizers (WGPs) as beamsplitters and is suitable for use at terahertz (THz) frequencies. The FPi was studied at the LUCX facility, KEK, Japan, and an $8 \mathrm{MeV}$ linear electron accelerator was used to generate coherent Smith-Purcell radiation. The FPi was designed to be easy to align and reposition for experiments at linear accelerator facilities. All of the components used were required to have a flat or well understood frequency response in the $\mathrm{THz}$ range. The performance of the FPi with WGPs was compared to that of a Michelson interferometer and the FPi is seen to perform well. The effectiveness of the beamsplitters used in the FPi is also investigated. Measurements made with the FPi using WGPs, the preferred beamsplitters, are compared to measurements made with the FPi using silicon wafers as alternative beamsplitters. The FPi performs well with both types of beamsplitter in the frequency range used $(0.3-0.5 \mathrm{THz})$. The successful measurements taken with the FPi demonstrate a compact and adaptable interferometer that is capable of analyzing $\mathrm{THz}$ radiation over a broad frequency range. The scheme is particularly well suited for polarization studies of $\mathrm{THz}$ radiation produced in an accelerator environment. Published by AIP Publishing. https://doi.org/10.1063/1.5018400

\section{INTRODUCTION}

The last few decades have seen increasing interest in electromagnetic radiation in the terahertz $(0.1-10 \mathrm{THz})$ frequency region, for both research and industrial applications ${ }^{1}$ and the increasing availability of $\mathrm{THz}$ radiation sources. ${ }^{2,3}$ The advancements in this field have led to a demand for experimental instrumentation and components that will work well in this frequency range. ${ }^{1}$ In this paper, the needs of those working with sub- $\mathrm{THz}$ or $\mathrm{THz}$ radiation generated in space-constrained accelerator environments are prioritized, but the work also has broader applications for any researchers looking to measure the frequency of $\mathrm{THz}$ radiation in a flexible and efficient manner.

An ongoing experimental study into the polarization of coherent Smith-Purcell radiation (cSPr) at the LUCX facility (KEK, Japan $)^{4,5}$ required an interferometer to make frequency measurements in the range 0.3-0.5 THz. cSPr is emitted when charged particles pass parallel to the surface of a periodic metal structure, such as a grating. cSPr is spatially distributed according to the dispersion relation given in the following equation:

$$
\lambda=\frac{p}{n}\left(\frac{1}{\beta}-\cos \theta\right)
$$

\footnotetext{
a)hannah.harrison@physics.ox.ac.uk

b) Now at National Physical Laboratory, Hampton Road, Teddington, Middlesex TW11 0LW, United Kingdom.
}

where $\lambda$ is the measured wavelength, $\theta$ is the observation angle (the angle between the direction of beam propagation and the position of a detector, see Fig. 1), $\beta=\frac{v}{c}$ is the normalized electron velocity, $p$ is the period of the grating, and $n$ is the diffraction order of the radiation (see Sec. III A). A consequence of this dispersion relation is that to measure a range of frequencies of cSPr, a range of observation angles is needed. This created the requirement that the interferometer be movable so that it could be placed into different angular positions between measurements. As the measurements were to be conducted over a range of frequencies, all components were required to be frequency independent or have a well understood frequency response within the operational range.

Working in a linear accelerator, it was necessary to consider the limited space and access typical to these environments. The size of the optical bench on which the interferometer was placed and moved around was limited which made it necessary for the design to be compact. As the accelerator is in a remote access tunnel, which cannot be entered while the accelerator is running, it was required that moving, re-aligning, and modifying the interferometer was a quick, accurate, and reproducible process. It also had to be remotely operable so that measurements could be taken while the accelerator was running.

This paper will describe a scheme that was designed to fulfill all of these prerequisites. Sections II-IV will discuss the type of interferometer built, the scheme developed for moving to different observation angles, and the beamsplitters chosen 

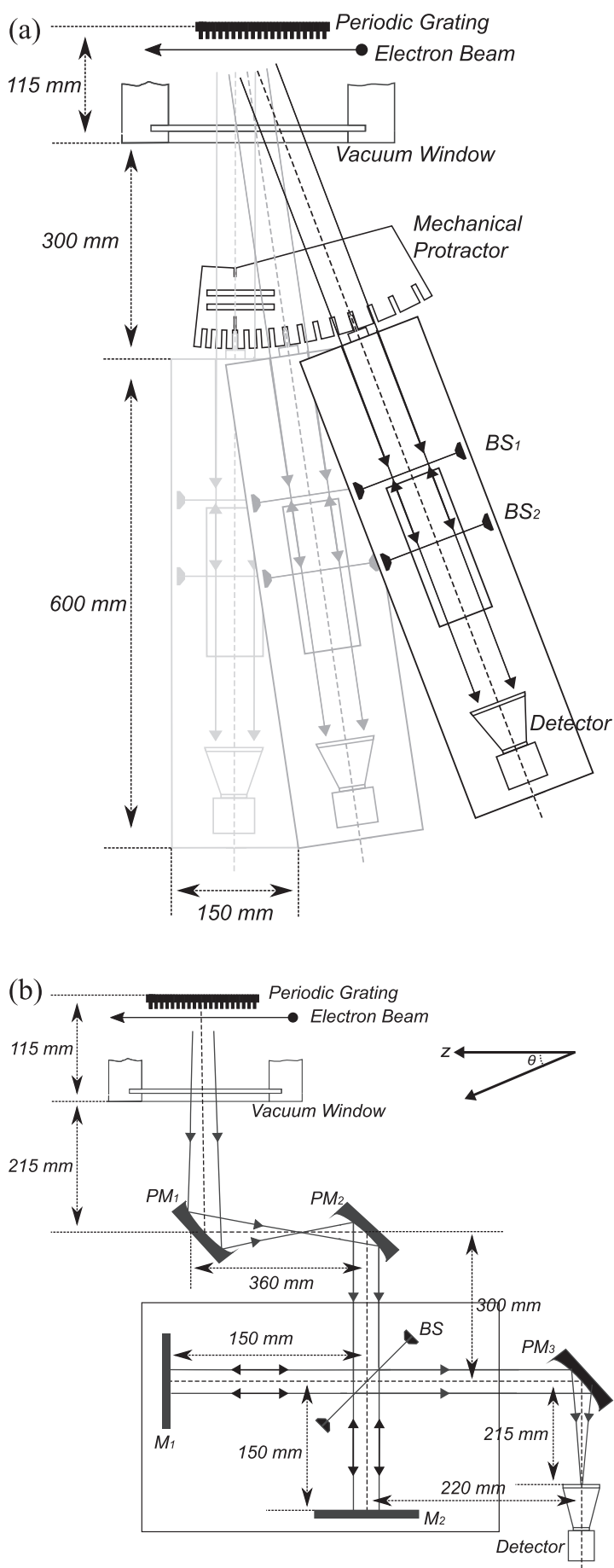

FIG. 1. Diagrams showing the layout of (a) the FPi as assembled at LUCX (where BS is a beamsplitter) and (b) the Mi used at LUCX (where PM is a parabolic mirror and $\mathrm{M}$ a plain mirror). $\mathrm{BS}_{2}$ and $\mathrm{M}_{1}$ are the components that are moved to create the varying path difference, respectively, and are positioned using motorized stages controlled by the accelerator control system. The definition of $\theta$ the observation angle, which is used in relation to both interferometer schemes, is shown with relation to $z$ the direction of the beam propagation.

for the frequency range. The analysis of a selection of these measurements will be presented in Sec. IV, including a comparison of the results for the new scheme with those taken with an existing Michelson interferometer (Mi) and a comparison of two different types of beamsplitter-freestanding wire grid polarizers (WGPs) and silicon wafers.

\section{DESIGN}

\section{A. Interferometer configuration}

The Fabry-Pérot interferometer (FPi) has been in use for over a hundred years. ${ }^{6,7}$ The FPi uses two partial reflectors to create a resonant cavity within which some of the light is trapped. Transmission is highest on, or close to, resonance and away from resonance, the device exhibits increased reflectivity and the transmission is reduced. The theory of the FPi at THz frequencies is well understood ${ }^{8}$ and there have been several experiments carried out using FPi's in the $\mathrm{THz}$ and sub- $\mathrm{THz}$ frequency ranges. ${ }^{9-14}$ The detrimental effect of incidentally creating Fabry-Pérot resonances by placing components with beamsplitting properties in the optical path has also been noted in this spectral region. ${ }^{15,16}$ Readers are directed to additional resources for an overview and history of the Fabry-Pérot interferometer ${ }^{17}$ and for details of the use of interferometers in the THz spectral region. ${ }^{18}$

The FPi was an attractive choice for the experimental configuration as it creates a linear optical path between the source and the detector which simplified the alignment of components compared to a setup with two axes (such as an Mi). The FPi requires two plane-parallel beamsplitters displaced along the optical axis allowing for the experimental setup to be moved to different observation angles without breaking the alignment. The full layout of the FPi instrument is shown in Fig. 1(a); the plate holding the FPi and detector covers an area of $600 \mathrm{~mm} \times 150 \mathrm{~mm}$ (length by width) on the optical bench such that the detector is located approximately $1 \mathrm{~m}$ away from the source of radiation. The design leaves space along the optical path for additional components if required, for example, lenses for collimation. In other experiments where space is very limited, the length could be further reduced. The FPi design includes a "mechanical" protractor that is used to align the whole detection system at varying angles with respect to the beamline. This kept the distance between the source and the detector constant for different observation angles. The uneven spacing of the mechanical protractor [Fig. 1(a)] allows for equal steps in detected frequency [of cSPr, see Eq. (1)] between each position. For a grating with a period of $0.70 \mathrm{~mm}$, the difference in frequency between each slot is $0.012 \mathrm{THz}$.

An alternative scheme based on a conventional Mi was also available at the LUCX facility [Fig. 1(b)]. A quick comparison of the diagrams of the two interferometers shows several key differences. First, the larger size and complexity of this Mi scheme makes it much more difficult to move and align at different positions on the optical bench. The Mi has three parabolic mirrors which were not included in the FPi design. The first two $\left(\mathrm{PM}_{1}\right.$ and $\left.\mathrm{PM}_{2}\right)$ are used to collimate the radiation and the last $\left(\mathrm{PM}_{3}\right)$ focuses the radiation onto the detector. The FPi and Mi schemes also have a different angular acceptance meaning that the frequencies measured with each will be slightly different. Finally, Fabry-Pérot and Michelson interferometers have different measurement properties. An Mi with a close to zero path difference, as shown in Fig. 1(b), is able to measure a broad range of frequencies, whereas an FPi can only measure a finite range of frequencies at once and so is not ideal when measuring broadband sources. ${ }^{17}$ 
(a)

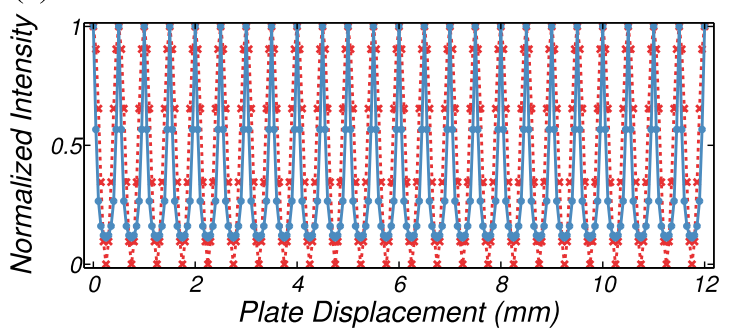

(b)

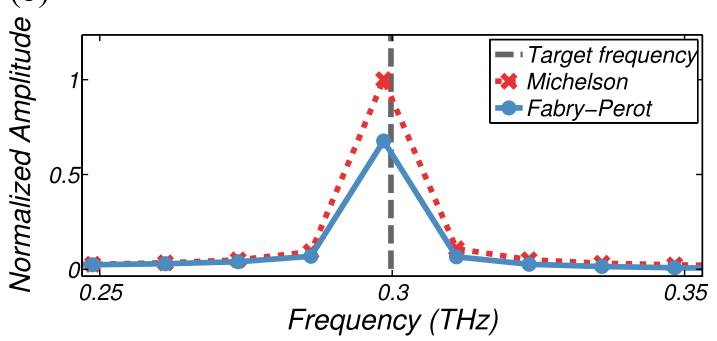

FIG. 2. Calculated (a) interferograms and (b) resulting frequency spectra that could be obtained using an FPi and an Mi to measure cSPr at $0.3 \mathrm{THz}$. The FPi in this simulation uses two ideal WGPs with a relative angular displacement of $45^{\circ}$.

Calculations were carried out to compare the expected interferograms from both the FPi and the Mi (see Fig. 2), both of these calculations assume a single frequency source $(0.3 \mathrm{THz})$ and ideal experiment with no noise or absorption. An ideal Michelson is in theory able to give perfect 0-1 modulation for a single frequency throughput as shown. By contrast, the degree of modulation observed from an FPi depends on the reflectivity of the beamsplitters used. ${ }^{19}$ For a system with a reflectivity less than 1 some baseline transmission is seen. The FPi described here used wire grid polarizers (WGPs) as beamsplitters (their use is discussed in detail in Sec. II B). The second beamsplitter has an angular displacement of $45^{\circ}$ with respect to the first beamsplitter which gives a reflectivity of 0.5 when using WGPs. Although the FPi has reduced modulation compared to the $\mathrm{Mi}$, the modulation is still pronounced and the frequency component remains easily distinguishable.

\section{B. Beamsplitters}

Figure 1(a) shows the positions of the two beam splitters $\left(\mathrm{BS}_{1}\right.$ and $\left.\mathrm{BS}_{2}\right)$ used to create the Fabry-Pérot cavity which provides the path difference in this interferometer. In the experiment discussed in this paper, it was advantageous to use freestanding WGPs as they have a flat response in the frequency range of interest and they were available from the measurements of the polarization of cSPr. Using the same components in both the frequency and polarization measurements reduced the overall number of components and allowed for quick changes between the two experimental configurations. WPGs are well established components used in a wide variety of settings. Their behavior is well understood theoretically and has been confirmed experimentally. ${ }^{20,21}$ To create a Fabry-Pérot cavity, it is necessary to rotate the second WGP with respect to the first WGP so that the wires of the two have an angle of $45^{\circ}$ between them. Increasing the angle between the two WGPs increases the reflectivity of the FPi and this in turn increases the modulation of the resultant interferogram, on the other hand, as the angle between the two WGPs becomes larger, the overall transmission of the system decreases. The best results require a compromise between the levels of modulation seen and the total throughput of the system; a relative angle of $45^{\circ}$ between the two WGPs took into account both of these factors. This angular displacement will split the radiation that has already passed through the first WGP into two equal components, one that is transmitted to the detector and the other that is reflected back into the FPi cavity. In Fig. 2(b), the interferogram and frequency spectrum are calculated assuming that the beamsplitters are WGPs with a $45^{\circ}$ relative offset.

WGPs are commonly used as passive components for selecting polarization in $\mathrm{THz}$ systems ${ }^{1,15,18}$ (including as beamsplitters for Martin-Puplett interferometers ${ }^{22}$ ) and as polarizing beamsplitters in the optical region. ${ }^{23}$ Various "one and two dimensional grids" have been used as beamsplitters for FPi's in the THz region previously. ${ }^{8}$ In particular, we draw attention to FPi designs using aluminum strips deposited on crystal quartz, ${ }^{13}$ copper wires glued to crystal quartz, polyethylene foil, or without substrate, ${ }^{14}$ and diffraction gratings ${ }^{24}$ as beamsplitters. There has also been discussion about the relative expense and difficulty of using WGPs as polarizing beamsplitters in the $\mathrm{THz}$ region. ${ }^{25}$ Commercially available freestanding WGPs appropriate for use up to $5 \mathrm{THz}$, are neither prohibitively expensive nor too fragile for use in an accelerator environment. In the absence of a support, their frequency response is largely governed by the interaction of the wire grid with the incident radiation ${ }^{20,21}$ and not by the frequency response of a substrate.

Tungsten wire polarizers with a wire thickness of $10 \mu \mathrm{m}$ and a wire periodicity of $30 \mu \mathrm{m}$ were installed in the locations indicated by $\mathrm{BS}_{1}$ and $\mathrm{BS}_{2}$ in Fig. 1(b). These WGPs are sold as suitable for use up to $3 \mathrm{THz} .{ }^{26}$ To illustrate the transmission properties of WGPs up to $1 \mathrm{THz}$, a calculation was carried out which is shown in Fig. 3. The model used for these calculations was developed in $1898^{27}$ and takes into account the periodicity of the wires and the thickness of the wires, but not any material properties. ${ }^{28}$ In Fig. 3(a), we see a gradual decrease in transmission over the frequency range of interest; however, at $1 \mathrm{THz}$ transmission is still above $99 \%$.

The beamsplitter used for the Mi shown in Fig. 1(a) was an n-doped silicon wafer. The behavior of doped silicon wafers can be modeled and the transmission properties can be calculated using the Cole-Davidson distribution (a modified Debye spectral response). ${ }^{29,30}$ The calculation of the transmission depends on several parameters including the density of the doping, the thickness of the wafer, and the angle of incidence of the radiation. In Fig. 4, the predicted response of an $\mathrm{n}$-doped silicon wafer of doping density $1 \times 10^{15} \mathrm{~cm}^{-3}$, of thickness $300 \mu \mathrm{m}$, and at an angle of incidence equal to $45^{\circ}$ is shown. The doping density and thickness are specific properties of the wafer used while the angle of incidence is due to the position of the beamsplitter in the Mi scheme as shown in Fig. 1(b). The beamsplitter efficiency is given, which is a measure of how perfectly a beamsplitter converts $s$ or $p$ polarized light 

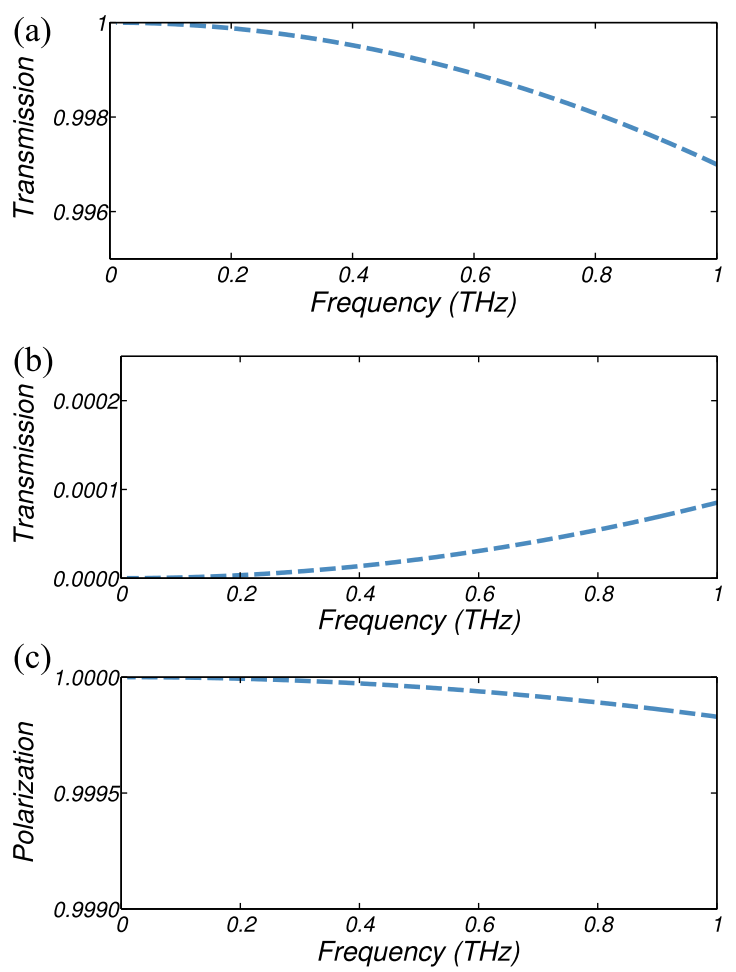

FIG. 3. Calculations showing the transmission of a WGP with a wire thickness of $10 \mu \mathrm{m}$ and wire periodicity of $30 \mu \mathrm{m}$. The transmission of incident radiation with electric field vector orientated (a) perpendicular and (b) parallel to the wire grids is shown. The (c) resulting polarization of the WGP across the frequency range.

into equally reflected and transmitted components. Here it is defined as $B E_{s / p}=4 R_{s / p} T_{s / p}$, (where $R$ is the reflectance and $T$ is the transmission). For both polarizations, the beamsplitter efficiency is a value between 0 and 1 . The $\mathrm{n}$-doped silicon wafer is shown to have a variable response over the range of frequencies used in the experiments. While this is not problematic for single narrowband frequency measurements, it could hinder experiments and the subsequent analysis if a range of frequencies is used.

High resistivity, $\rho \approx 5 \mathrm{k} \Omega \mathrm{cm}$, silicon wafers were also used as beamsplitters in the FPi as part of this study. They were

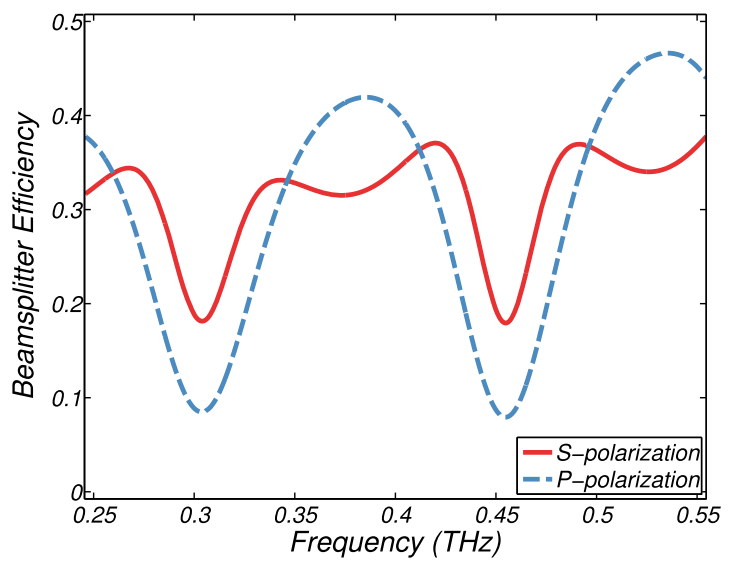

FIG. 4. Beamsplitter efficiency of the n-doped silicon wafer used in the Mi. The calculation was done for a $300 \mu \mathrm{m}$ thick, n-doped silicon wafer $\left(\mathrm{n}=1 \times 10^{15} \mathrm{~cm}^{-3}\right)$ with an incidence angle of $45^{\circ}$.

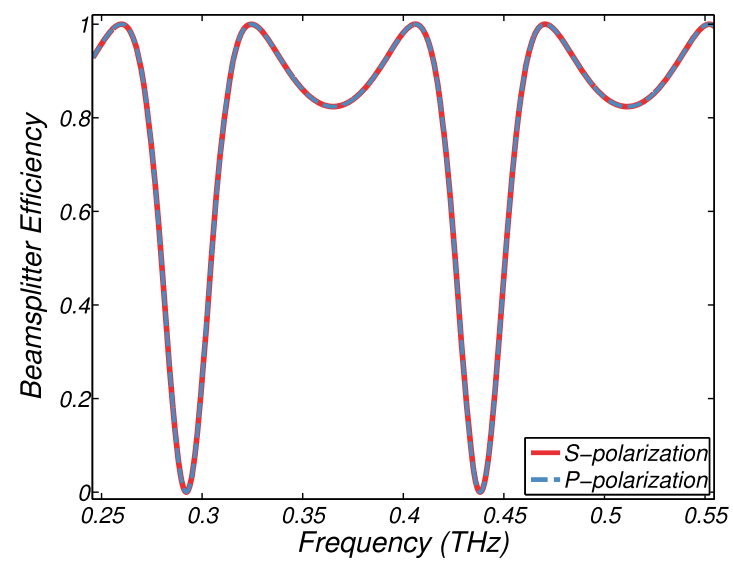

FIG. 5. Beamsplitter efficiency of a high resistivity silicon wafer used in the FPi. The calculation was done for a $300 \mu \mathrm{m}$ thick, $\rho \approx 5 \mathrm{k} \Omega \mathrm{cm}$ silicon wafer with an incidence angle of $0^{\circ}$.

used to examine the behavior of the FPi with an alternative type of beamsplitter that had a different frequency response. The transmission properties of the wafers used are shown in Fig. 5. The beamsplitter efficiency of a high resistivity wafer of thickness $300 \mu \mathrm{m}$ at an angle of incidence equal of $0^{\circ}$ is shown. The figure considers the beamsplitter efficiency of one wafer although it should be noted that two were used to form the Fabry-Pérot cavity. Similar to the n-doped silicon wafer, a frequency dependent response is seen across the frequency range of interest. We note that in this case, the beamsplitter efficiency is polarization independent with the result that the $\mathrm{s}$ and $\mathrm{p}$ components are equal. As in this scheme the incoming radiation is at normal incidence with respect to the silicon wafers, the FPi is not vulnerable to walk-off effects.

The silicon wafers used in both the Mi and FPi schemes are seen to exhibit highly variable beamsplitter efficiency across the frequency range of interest in this study. The calculated response is predicted to result in variations in transmission across this range. This presents difficulties for any experiment where it is useful to compare the intensities of radiation measured at different frequencies. It should not, however, strongly affect the frequency components of the transmitted radiation in narrowband measurements.

\section{EXPERIMENTAL CONFIGURATION}

\section{A. cSPr generation at LUCX}

The FPi design described was developed in order to conduct studies into the polarization properties of cSPr as a

TABLE I. LUCX, beam parameters.

\begin{tabular}{lc}
\hline \hline Parameter & Expected values \\
\hline Beam energy, typ (Mev) & 8 \\
Charge per bunch, typ (pC) & 50 \\
Bunch length (ps) & $0.15-10$ \\
Repetition rate, max $\left(\mathrm{s}^{-1}\right)$ & 12.5 \\
Normalized emittance $\left(\epsilon_{x} \times \epsilon_{y}\right)(\mathrm{mm} \mathrm{mrad})$ & $4.7 \times 6.5 \pi$ \\
\hline \hline
\end{tabular}



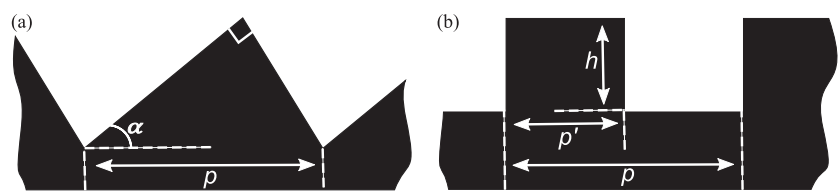

FIG. 6. Grating profiles showing one facet of (a) sawtooth and (b) strip gratings. The experiments described in this paper used two sawtooth gratings with periodicity $(p)$ of $0.70 \mathrm{~mm}$ and $1.00 \mathrm{~mm}$, both with a blaze angle $(\alpha)$ equal to $30^{\circ}$. The strip grating used had a periodicity $(p)$ of $0.70 \mathrm{~mm}$, an equal ratio between the extruding and non-extruding section $\left(p^{\prime}=\frac{p}{2}\right)$ and a facet depth (h) of $0.25 \mathrm{~mm}$.

function of frequency. The frequency of the radiation detected at each position was measured to confirm the presence of $\mathrm{cSPr}$ and to check for errors in the experimental setup. The properties of the LUCX linear accelerator (KEK, Japan) and its beam parameters are given in Table I; more information on this accelerator system and recent experiments conducted at LUCX is available in the literature. ${ }^{31-34}$

cSPr is produced when charged particles travel above a periodic metallic grating. ${ }^{35}$ The radiation is spatially distributed according to the dispersion relation given in Eq. (1). The properties of cSPr had been extensively discussed in a variety of publications. ${ }^{35-37}$ As its spectral properties are well understood, cSPr was used here to study the performance of the FPi with WGPs. Three gratings were used (i) $1.00 \mathrm{~mm}$ period sawtooth grating, (ii) $0.70 \mathrm{~mm}$ period sawtooth grating, and (iii) $0.70 \mathrm{~mm}$ period strip grating. These grating profiles are shown in Fig. 6. All the gratings were of the same dimension, $20 \mathrm{~mm} \times 60 \mathrm{~mm}$ (width $\times$ length), with their long axis placed parallel to the electron beam. Each grating was placed in the vacuum chamber in turn adjacent to the electron beam and the emitted cSPr was extracted via a sapphire window (as shown in Fig. 1). It was possible to access the range of angles from $85^{\circ}$ to $118^{\circ}$ with the FPi. The limitations on the angles available were due to the finite dimensions of the window and restricted space on the bench. Given the periodicities of the gratings used and the Smith-Purcell dispersion relation [Eq. (1)], the frequencies emitted in this angular range are $0.203-0.465 \mathrm{THz}$. The frequencies that can be detected are further constrained by the range of the detector, which in these experiments was a zero bias Schottky barrier diode with a detection range from $0.325 \mathrm{THz}$ to at least $0.500 \mathrm{THz}^{38}$ and a lower cut-off frequency of $0.268 \mathrm{THz}^{39}$

\section{B. Interferometer operation}

Two key properties of the interferometer are the length of the scan $x_{l}$ (the total distance that the movable part travels) and

TABLE II. Interferometer comparison.

\begin{tabular}{lcc}
\hline \hline & Michelson & Fabry-Pérot \\
\hline$x_{l}(\mathrm{~mm})$ & $\approx 11$ & $\approx 11$ \\
$\Delta x(\mathrm{~mm})$ & 0.05 & 0.1 \\
$f_{s}(\mathrm{THz})$ & 3.0 & 1.5 \\
$f_{N q}(\mathrm{THz})$ & 1.5 & 0.75 \\
$\Delta f(\mathrm{THz})$ & $\approx 0.0135$ & $\approx 0.0135$ \\
\hline \hline
\end{tabular}

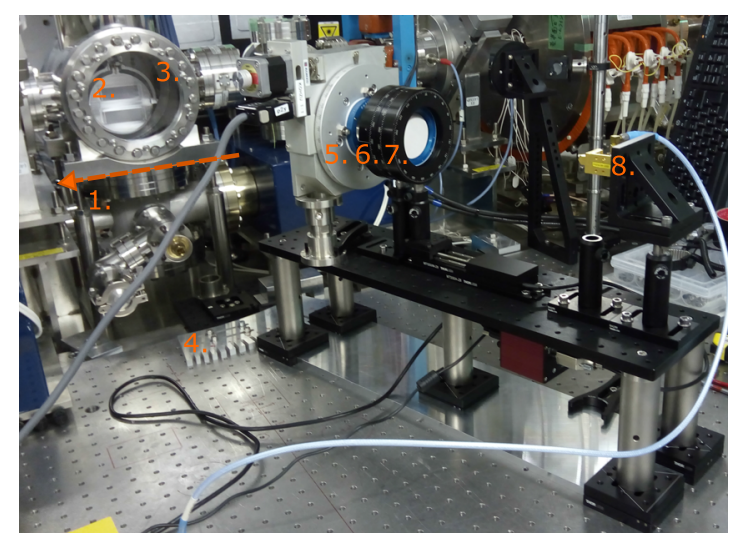

FIG. 7. FPi installed on the optical bench at the LUCX facility. The components are as follows: 1 . electron beam trajectory, 2 . periodic grating, 3 . vacuum window, 4. mechanical protractor, 5. WGP on rotation mount, 6. BS $1,7 . \mathrm{BS}_{2}$, 8. zero bias Schottky barrier diode detector. The first WGP (5.) is aligned with $B S_{1}$ and does not affect the frequency measurement.

the interval between each measurement $\Delta x$. These two properties determine the resolution and the range of the frequency spectrum that is calculated from the interferogram

$$
\begin{aligned}
f_{s} & =\frac{c}{2 \Delta x}, \\
\Delta f & =\frac{c}{2 x_{l}} .
\end{aligned}
$$

Equation (2) shows that the sampling frequency of the system $\left(f_{s}\right)$ which is inversely proportional to the measurement interval $(\Delta x)$. Equation (3) shows that the frequency resolution $(\Delta f)$ is inversely dependent on the scan length $\left(x_{l}\right) \cdot{ }^{40} \mathrm{In}$ both Eqs. (2) and (3), the speed of light is denoted by $c$. When using discrete Fourier transforms (such as FFTs) to calculate the measured frequency spectra, the maximum resolvable frequency is defined by the Nyquist frequency $\left(f_{N q}\right)$, as shown in the following equation:

$$
f_{N q}=\frac{f_{s}}{2}=\frac{c}{4 \Delta x} .
$$

Table II shows the parameters used in experiments with the Michelson and Fabry-Pérot interferometers. While the length of the scans $x_{l}$ is the same for both, the stepsize $\Delta x$ used for the Michelson is a factor of two smaller. The stepsize used for the FPi studies was increased and the maximum resolvable frequency of $0.75 \mathrm{THz}$ was considered to be sufficient for this study.

Figure 7 shows a photograph of the FPi installed on the optical bench. An additional polarizer was added during the experiment, between the sapphire vacuum window and the first polarizer of the Fabry-Pérot cavity $\left(B S_{1}\right)$, to facilitate studies of the polarization properties of the $\mathrm{cSPr}$. The wires of the extra polarizer were aligned with the wires of $B S_{1}$ during all interferogram measurements. While this extra component will cause a reduction in signal intensity, it did not affect the frequency measurement.

\section{RESULTS AND DISCUSSION}

In this section, we bring together results obtained using the Mi and FPi interferometer schemes at the LUCX facility. These 
results were obtained over a series of experimental runs during which it is possible that some of the accelerator parameters may have varied and this may have affected the generation of cSPr. Changes to parameters such as the beam position, average charge, and the emittance of the bunch would lead to variation in the intensity of cSPr generated at a given frequency but would not have impacted the frequency content reaching the detector. The results presented are used to compare the frequency content measured by the interferometer schemes but do not compare the intensity of radiation measured by each scheme. For this reason, all the frequency spectra shown have been normalized, avoiding any dependence on the beam parameters and accelerator setup.

The FPi was used to measure the cSPr generated by the three gratings. Examples of spectra generated at different angles by the FPi (with WGPs as beamsplitters) are shown in Fig. 8. The graph shows results using cSPr generated by a sawtooth grating profile with a period of $0.70 \mathrm{~mm}$. Frequency spectra have been observed at a set of angles $\left(\theta=107.8^{\circ}, 95.7^{\circ}\right.$, and $88.4^{\circ}$ ) and the measured frequencies correspond well to the theoretical values given by Eq. (1).

In each of the three frequency spectra, we can see the points generated by the FFT, with errorbars and a Gaussian fit. The errorbars are calculated based on the statistical uncertainty provided by the raw data from the interferograms. The raw FFT can give us a peak frequency, with uncertainty determined by the resolution of the FFT. The amplitude of each frequency spectrum has been scaled so that the highest data point in the displayed frequency range is equal to one.

The FPi and Mi were used to measure the frequency of cSPr generated by the $1.00 \mathrm{~mm}$ sawtooth grating. The results are used to compare the operation of these two interferometers. Figure 9 shows the measured peak frequenciesgiven by the maximum point of each frequency spectrumfor both interferometers as well as the expected cSPr frequency at all positions [calculated using Eq. (1)]. As the Mi could not be rotated without affecting the alignment, the only

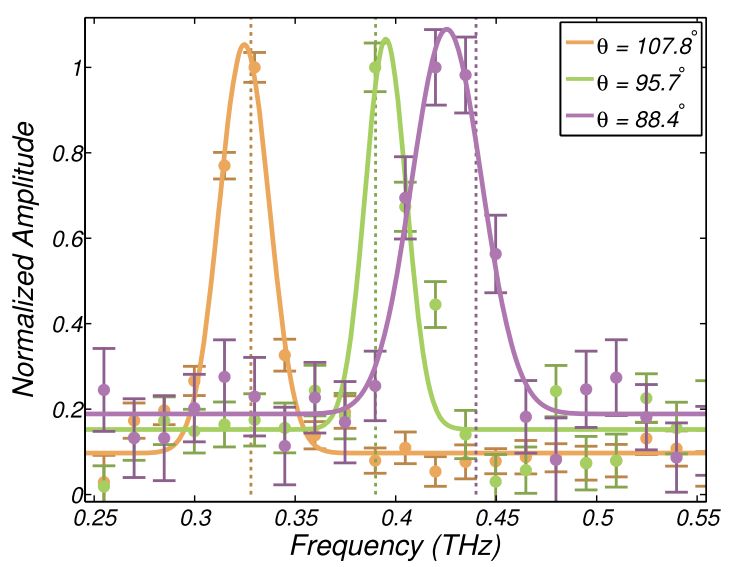

FIG. 8. A range of frequency spectra taken at different angular positions with the FPi. The measured frequency spectra are plotted along with a corresponding Gaussian fit (solid lines). The cSPr was generated using the $0.70 \mathrm{~mm}$ period sawtooth grating. Using the dispersion relation of cSPr [Eq. (1)], the expected frequencies of the three angular positions- $107.8^{\circ}, 95.7^{\circ}$, and $88.4^{\circ}$ - can be calculated to be $0.33,0.39$, and $0.44 \mathrm{THz}$, respectively. The dashed vertical lines indicate the expected frequencies for the measured spectra.

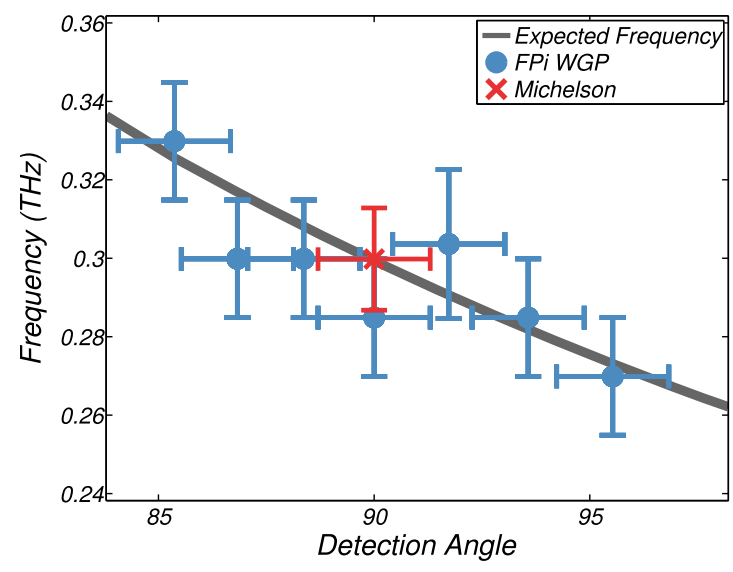

FIG. 9. Experimental results for the FPi and Mi measuring cSPr at LUCX. The theoretical prediction of the cSPr frequency is shown by the gray line. The frequency is given by the peak value of the frequency spectrum and the errors are determined by the resolution of the frequency spectrum and the angular uncertainty of the mechanical protractor.

measurements conducted with it correspond to the observation angle $\theta=90^{\circ}$. A range of frequency measurements taken with the FPi at different observation angles are also given. The error due to the finite angular alignment of the FPi is also considered. It is estimated that the mechanical protractor allows deviation from the designed angular position of up to $\pm 1.3^{\circ}$ leading to possible frequency deviations of up to \pm 0.01 THz. A similar uncertainty on the peak frequency is obtained using both interferometers $(\approx 0.015 \mathrm{THz})$ and both sets of frequency measurements are consistent with the predicted values.

In Fig. 10, the results for both interferometers measuring cSPr generated by the $1.00 \mathrm{~mm}$ period sawtooth grating at observation angle $\theta=90^{\circ}$ are given in more detail. In Fig. 10(a), the interferograms are shown and they have clear similarities with the idealized operation predictions shown in Fig. 2(a). The FPi shows reduced intensity modulation compared to the $\mathrm{Mi}$, and the spectral plot, Fig. 10(b), shows that both interferometers have a peak frequency close to the expected $0.3 \mathrm{THz}$ value. The lower signal to noise ratio seen for the FPi spectrum is due to the reduced intensity of the radiation measured compared to that measured by the Mi. As previously discussed, the relative angle between the two WGPs used as beamsplitters in the FPi scheme was a compromise between achieving high levels of modulation and high overall throughput of the system. Changes to the accelerator parameters in the interval between the two sets of measurements could have also affected both the intensity of cSPr and the noise level measured. Gaussian fits have been plotted alongside the raw FFT in order to quantify how broad the peaks measured by each scheme are and to get an improved central frequency estimate. The Gaussian fitting gives us $\mu_{F P i}=0.289 \mathrm{THz}, \sigma_{F P i}=0.015 \mathrm{THz}$ and $\mu_{M i}=0.300 \mathrm{THz}, \sigma_{M i}=0.007 \mathrm{THz}$. From these parameters, it is clear that both designs are able to measure accurately the frequency, and that the FFT produced from the interferogram taken with the FPi has a broader peak. This could be due to the larger angular acceptance of the FPi system, imperfect alignment of the two beamsplitters, or a combination of both. The angular acceptance of the FPi scheme was $\pm 2.5^{\circ}$, the 

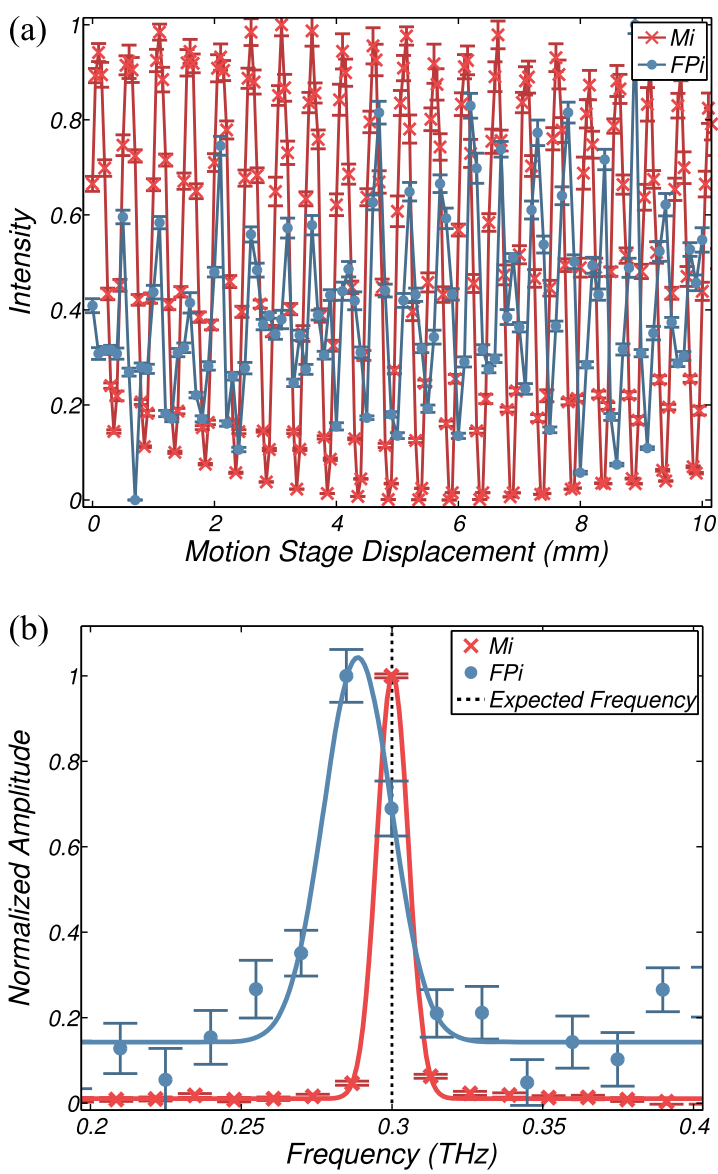

FIG. 10. Results using the FPi and the Mi to measure the frequency of cSPr generated by $1.00 \mathrm{~mm}$ period sawtooth grating and observed at $90^{\circ}$. Both (a) the interferogram and (b) the resultant frequency spectrum are shown for each interferometer. Solid lines in (a) are a guide to the eye and in (b) are a Gaussian fit to the peaks. The expected frequency of $0.30 \mathrm{THz}$ is shown by the dashed vertical line in (b). The amplitudes of both frequency spectra have been scaled so that the highest data point in the displayed frequency range is equal to one.

frequency range that this allows varies based on the periodicity of the grating $(p)$ and on the angle of observation $\theta$ - see Eq. (1). For the range of $\theta$ and $p$ used, it is on the order of $0.01 \mathrm{THz}$. Specifically for $p=0.7 \mathrm{~mm}$ at and $\theta=90^{\circ}$, frequencies transmitted are in the range $0.300 \pm 0.013 \mathrm{THz}$. The angular acceptance of the Mi scheme is determined by the parabolic mirror $\left(P M_{1}\right)$ as shown in Fig. 1(b). The angular acceptance of either scheme could easily be modified if it was required.

Measurements were also made with the FPi using both WGPs and silicon wafers as beamsplitters. These were taken over a range of observation angles, and using two of the available gratings to generate the cSPr. Results taken using the $0.7 \mathrm{~mm}$ period strip and sawtooth gratings are shown in Figs. 11(a) and 11(b), respectively. The studies were carried out using the same accelerator parameters and the same FPi layout.

The measured frequencies are compared to the expected frequencies for cSPr at all observation angles. The frequencies measured for both types of beamsplitter are close to the expected value of cSPr from theory. This suggests that the performance of the FPi is unaffected by changing from silicon wafers to WGPs. There is little difference in the results for the
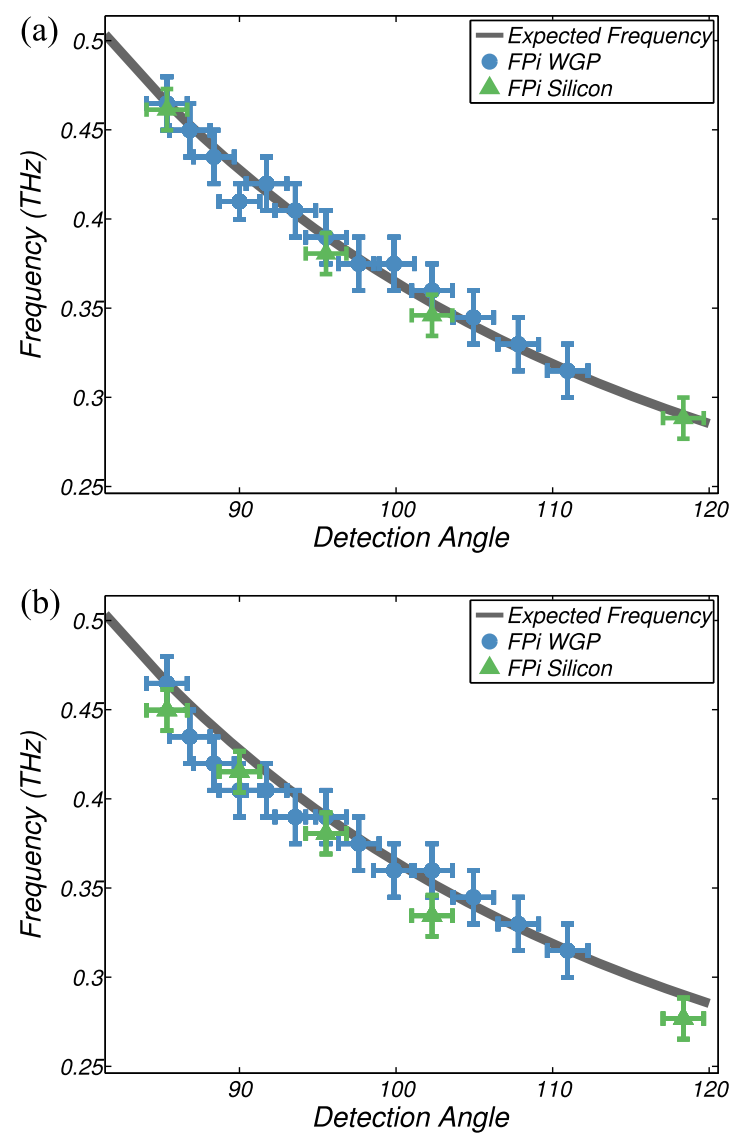

FIG. 11. Experimental results for the FPi with different beam splitters (silicon wafers and WGPs) measuring cSPr over a range of observation angles. The cSPr was generated using a (a) strip grating and a (b) sawtooth grating, both with a periodicity of $0.70 \mathrm{~mm}$. The theoretical prediction of the frequency of cSPr, Eq. (1), is indicated by the smooth lines. Frequency points plotted are determined by the peak of the spectrum in each case. The errors shown are determined by the resolution of the frequency spectrum and the angular uncertainty of the mechanical protractor.

strip and sawtooth gratings (as expected), demonstrating the consistency of the scheme.

In order to study these results in more depth, the frequency spectra measured at observation angle $95.7^{\circ}$, with $\mathrm{cSPr}$ generated by the $0.70 \mathrm{~mm}$ sawtooth grating, using the FPi with both WGPs and silicon wafers are compared in Fig. 12. In Fig. 5, a calculation is presented which shows that the beamsplitter efficiency of the high resistivity silicon wafers is expected to vary significantly across the frequency range. Readers will note that the expected frequency for this measurement $(0.39 \mathrm{THz})$ avoids the most dramatic modulations, so the silicon beamsplitter can be expected to perform well at this position. It is clear from this figure that consistent frequency measurements are achieved with the two different types of beamsplitter. The variations observed are likely due to changes in the alignment of the beamsplitters in the FPi. Gaussian fitting gives us $\mu_{W G P}=0.395 \mathrm{THz}, \sigma_{W G P}=0.016$ $\mathrm{THz}$ and $\mu_{\text {sili }}=0.379 \mathrm{THz}, \sigma_{\text {sili }}=0.016 \mathrm{THz}$. The central frequencies measured are in agreement within the resolution of the frequency spectra and the alignment error. The peaks of both spectra have a similar width, which suggests that the beamsplitters do not have a strong effect on the frequency resolution. 


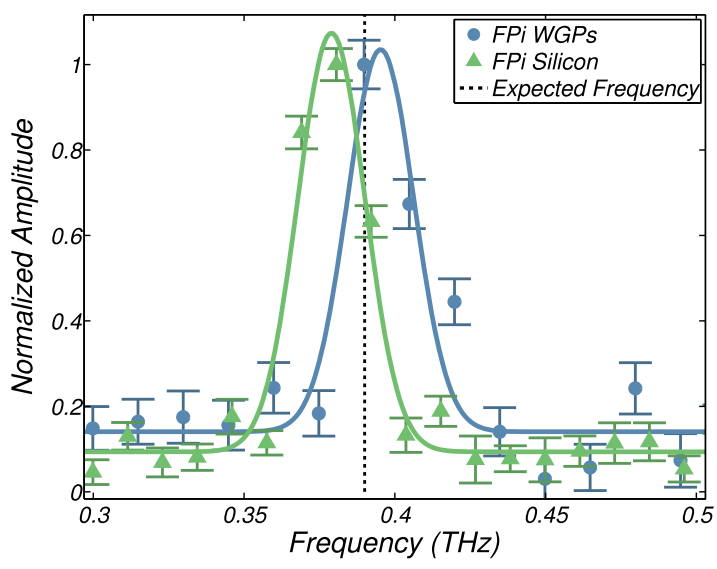

FIG. 12. Results using the FPi with WGPs and silicon wafers as beamsplitters to measure the frequency of cSPr generated by the $0.70 \mathrm{~mm}$ period sawtooth grating and observed at $95.7^{\circ}$. The solid lines are Gaussian fits of the frequency spectra. The expected frequency of $0.39 \mathrm{THz}$ is shown by the dashed vertical line in (b).

\section{CONCLUSIONS}

A Fabry-Pérot interferometer was designed, built, and demonstrated to be capable of carrying out frequency measurements of cSPr in the $\mathrm{THz}$ region. It was required as part of an ongoing investigation into the polarization properties of cSPr and needed to be compact, movable, and easy to modify in order to take other measurements. These requirements are satisfied by the design shown in Fig. 1(a). The use of WGPs as beamsplitters was very convenient as they formed a crucial part of the polarization study. They are shown to be highly appropriate for use as beamsplitters due to their flat response at the frequencies of interest (particularly when compared to the responses of silicon wafers). The device was built, tested, and used in the typical accelerator environment of the LUCX facility.

Comparative studies of the FPi and Mi schemes were carried out using cSPr. The results showed that the FPi has comparable performance to an $\mathrm{Mi}$, with both schemes measuring frequencies close to the expected values. The FPi was also demonstrated to function well for both of the beamsplitters used. Measurements taken using both silicon wafers and WGPs as beamsplitters were compared and the two sets of measurements were shown to be very similar. Repetition of some of these measurements in order to make a comparison of the intensity of the cSPr as well as the frequency content would be a desirable extension to this study.

The FPi has achieved its requirement of taking frequency measurements whilst remaining flexible, compact, and portable. The authors believe that this approach will be applicable and beneficial to other experimental setups, in particular, studies which are concerned with $\mathrm{THz}$ radiation emitted in accelerator-like environments, and also believe that this method may be of interest to a wider audience.

\section{ACKNOWLEDGMENTS}

This work was supported by the UK Science and Technology Facilities Council (STFC UK) through Grant No.
ST/M003590/1, the Leverhulme Trust through the International Network Grant (No. IN-2015-012), the Photon and Quantum Basic Research Coordinated Development Program from the Ministry of Education, Culture, Sports, Science and Technology, Japan and JSPS KAKENHI Nos. 23226020 and 24654076. H. Harrison would like to thank STFC UK (No. ST/M50371X/1) and John Adams Institute of Accelerator Science (University of Oxford) for supporting their DPhil project. M. Shevelev acknowledges the Competitiveness enhancement program of Tomsk Polytechnic University.

${ }^{1}$ S. S. Dhillon, M. S. Vitiello et al., J. Phys. D: Appl. Phys. 50, 043001 (2017).

${ }^{2}$ G. P. Williams, Rep. Prog. Phys. 69, 301 (2006).

${ }^{3}$ K. Reimann, Rep. Prog. Phys. 70, 1597 (2007).

${ }^{4} \mathrm{H}$. Harrison et al., in Proceedings of the 7th International Particle Accelerator Conference (IPAC'16), Busan, Korea, May 8-13, 2016 (JACoW, 2016), pp. 340-343.

${ }^{5} \mathrm{H}$. Harrison et al., in Proceedings of the 5th International Beam Instrumentation Conference (IBIC'16), Barcelona, Spain, September 13-18, 2016 (JACoW, 2017), pp. 472-475.

${ }^{6}$ C. Fabry and A. Pérot, Ann. Chim. Phys. 12, 459 (1897).

${ }^{7}$ C. Fabry and A. Pérot, Ann. Chim. Phys. 16, 115 (1899).

${ }^{8}$ E. Baker and B. Walker, J. Phys. E: Sci. Instrum. 15, 25 (1982).

${ }^{9}$ D. Jahn, A. Soltani et al., in International Conference on Infrared, Millimeter, Terahertz Waves, IRMMW-THz, November 1, 2016, 2017.

${ }^{10}$ G. Doucas, M. F. Kimmitt et al., Phys. Rev. Spec. Top.-Accel. Beams 5, 14 (2002).

${ }^{11}$ A. Doria, G. P. Gallerano, E. Giovenale, G. Messina, and G. Doucas, Nucl. Instrum. Methods Phys. Res., Sect. A 483, 263 (2002).

${ }^{12}$ R. Braakman and G. A. Blake, J. Appl. Phys. 109, 063102 (2011).

${ }^{13}$ T. Hori, T. Matsui, K. Araki, and H. Inomata, Opt. Lett. 14, 302 (1989).

${ }^{14}$ R. Ulrich, K. F. Renk, and L. Genzel, IEEE Trans. Microwave Theory Tech. 11, 363 (1963).

${ }^{15}$ A. Ferraro, D. C. Zografopoulos et al., Opt. Lett. 41, 2009 (2016).

${ }^{16}$ A. Isozaki, T. Kan et al., in 2013 Transducers Eurosensors XXVII: The 17th International Conference on Solid-State Sensors, Actuators and Microsystems (IEEE, 2013), pp. 530-533.

${ }^{17}$ W. H. Steel, "Multiple-beam interferometers," in Interferometry, 2nd ed. (Cambridge University Press, Cambridge, United Kingdom, 1983), Chap. 9.3. The Fabry-Pérot interferometer.

${ }^{18}$ M. Naftaly, "Terahertz optics," in Terahertz Metrology (Artech House, Boston, 2015).

${ }^{19}$ E. Hecht, Optics, 4th ed. (Pearson Education, Inc., Publishing as Addison Wesley, San Francisco, CA, USA, 2002).

${ }^{20}$ G. R. Bird and M. Parrish, Jr., J. Opt. Soc. Am. 50, 886 (1960).

${ }^{21}$ P. Yeh, Opt. Commun. 26, 289 (1978).

${ }^{22}$ W. Liu, D. Wu et al., Nucl. Instrum. Methods Phys. Res., Sect. A 614, 313 (2010).

${ }^{23}$ M. Xu, H. Urbach, D. K. G de Boer, and H. J. Cornelissen, Opt. Express 13 , 2303-2320 (2005); H. Raether, Surface Plasmons on Smooth and Rough Surfaces and on Gratings, Optical Liquid Crystal Displays (Springer, 1988).

${ }^{24}$ V. Y. Balakhanov, V. K. Zhibotov, and A. V. Titov, J. Appl. Spectrosc. 11, 831 (1969).

${ }^{25}$ C. W. Berry and M. Jarrahi, J. Infrared, Millimeter, Terahertz Waves 33, 127 (2012).

${ }^{26}$ Purewave Polarizers, "10 micron wire grid polarizer: PW010-030-075," http://www.purewavepolarizers.com/wire-grid-polarizers (2017) [Online; accessed 14 August 2017].

${ }^{27}$ H. Lamb, Proc. London Math. Soc. 29, 523 (1898)

${ }^{28}$ T. Larsen, IRE Trans. Microwave Theory Tech. 10, 191 (1962).

${ }^{29}$ T.-I. Jeon and D. Grischkowsky, Phys. Rev. Lett. 78, 1106 (1997).

${ }^{30}$ T.-I. Jeon and D. Grischkowsky, Appl. Phys. Lett. 72, 2259 (1998).

${ }^{31}$ M. Fukuda, S. Araki et al., Nucl. Instrum. Methods Phys. Res., Sect. A 637, S67 (2011).

${ }^{32}$ A. Aryshev, M. Shevelev, Y. Honda, N. Terunuma, and J. Urakawa, Appl. Phys. Lett. 111, 033508 (2017); e-print arXiv:1507.00330.

${ }^{33}$ G. Naumenko, A. Aryshev et al., Nucl. Instrum. Methods Phys. Res., Sect. B 402, 153 (2017).

${ }^{34}$ M. Shevelev, A. Aryshev et al., Nucl. Instrum. Methods Phys. Res., Sect. A 771, 126 (2015).

${ }^{35}$ J. Brownell, J. Walsh, and G. Doucas, Phys. Rev. E 57, 1075 (1998). 
${ }^{36}$ A. S. Kesar, Phys. Rev. Spec. Top.-Accel. Beams 13, 022804 (2010).

${ }^{37}$ D. V. Karlovets and A. P. Potylitsyn, Phys. Rev. Spec. Top.-Accel. Beams 9, 080701 (2006)

${ }^{38}$ Virginia Diodes, "Zero bias detectors-VDI model: WR2.2ZBD," http:// vadiodes.com/index.php/en/products/detectors?id=122/ (2016) [Online; accessed 21 April 2016].
${ }^{39}$ Virginia Diodes, "Virginia Diodes, Inc., waveguide band designations," http://vadiodes.com/VDI/pdf/waveguidechart200908.pdf (2016) [online; last modified 29 June 2010].

${ }^{40}$ G. W. F. Drake, "Infrared spectroscopy," in Springer Handbook of Atomic, Molecular, and Optical Physics (Springer, New York, 2006), Chap. 40.4.3. The operation of spectrum determination. 